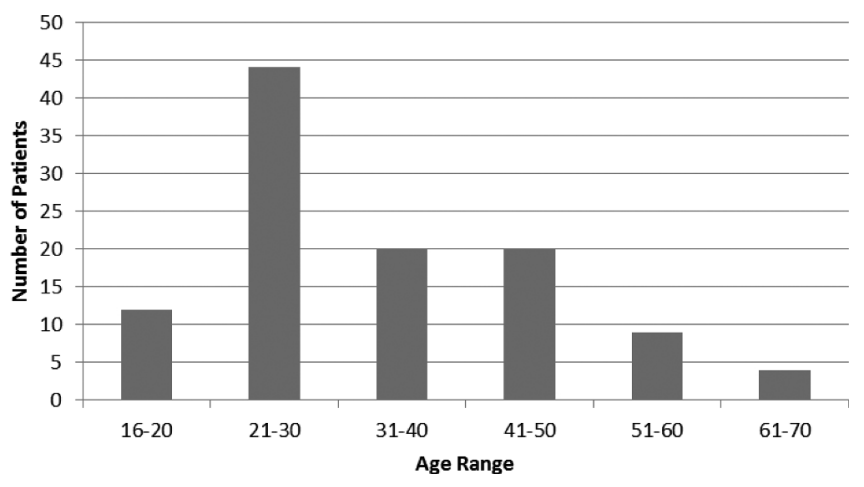

Abstract 72 Figure 1 Age of T21 cohort: NORPAP database

the highest mortality. $1 / 3$ of all patients had PAH and 1/4 of these died during follow up. Survival into the $4^{\text {th }}$ and $5^{\text {th }}$ decade is frequent. Patients in the $3^{\text {rd }}$ decade now may expect to survive for another 25-30 years. Ongoing cardiac and noncardiac morbidity requires dedicated CHD clinics, frequently with PAH clinicians given that $50 \%$ of those under 40 have not had surgery and may need targeted therapy.

\section{MATERNAL AND FETAL OUTCOMES IN WOMEN WITH AORTOPATHY: EXPERIENCE IN A TERTIARY JOINT CARDIAC OBSTETRIC CENTRE}

${ }^{1}$ Emma Plunkett, ${ }^{2}$ Suzanna Bull, ${ }^{2}$ Ruth Gertig, ${ }^{3}$ Sarah Bowater, ${ }^{3}$ Paul Clift, ${ }^{3}$ Lucy Hudsmith, ${ }^{3}$ Sara Thorne*, ${ }^{2}$ Katie Morris, 'Tara Selman, 'Peter Thompson. 'Birmingham Women's Hospital; ${ }^{2}$ University of Birmingham; ${ }^{3}$ University Hospitals Birmingham; *Presenting Author

\subsection{6/heartjnl-2016-309890.73}

Introduction Pregnancy is associated with increased risk of cardiovascular complication for women with aortic pathology. The University Hospitals Birmingham (UHB) and Birmingham Women's Hospital (BWH) joint cardiac obstetric service sees women with aortopathy for pre-pregnancy assessment and counselling and subsequently manages them during pregnancy. A $10 \%$ incidence of serious maternal complications during pregnancy is widely quoted. We sought to review our recent cases and describe maternal and fetal outcomes in our patient population.

Methods Female patients aged 16-35 with aortopathy and a recent pregnancy (2008 $\dddot{\mathrm{i} c} 1 \frac{1}{2}$ 2015) were identified from the UHB database. Patients with a bicuspid aortic valve, structural congenital cardiac disease and coarctation of the aorta were excluded. Electronic and paper patient records and imaging results were reviewed to collect data regarding diagnosis, medical management, aortic dimensions, maternal obstetric and cardiovascular complications during or subsequent to pregnancy (within 1 year) and fetal outcome.

Results 23 patients were identified with the following diagnoses: Marfan syndrome $(\mathrm{n}=14)$, Ehlers-Danlos $(\mathrm{n}=4)$, Loeys-Dietz $(n=1)$ and undefined $(n=4)$ aortopathy. There were no patients with Turner syndrome within this cohort. One patient was excluded as she was under follow up for a family history of aortic disease which did not subsequently manifest itself in the patient. There were a total of 28 pregnancies. Beta blockers were prescribed in 21 pregnancies. Magnetic resonance imaging (MRI or MRA) was performed in 17 pregnancies.

Maternal outcomes Two patients had aortic dissections related to pregnancy. One was diagnosed immediately post partum in a woman with known Marfan syndrome and the other at 38 weeks gestation in a woman subsequently found to have to have Marfan syndrome. Both had successful emergency aortic root replacement, the second immediately after delivery of the fetus by caesarean section. One patient with undefined aortopathy presented acutely with chest pain and a rapidly dilating aorta; she had an emergency aortic root replacement at 22/40 gestation and was found to have a necrotising granulomatous aortitis. No patients died. Two patients had a change in aortic dimensions noted during pregnancy.

Fetal outcomes Two of the pregnancies resulted in therapeutic abortions related to occurrence of inherited lesions in the fetus.

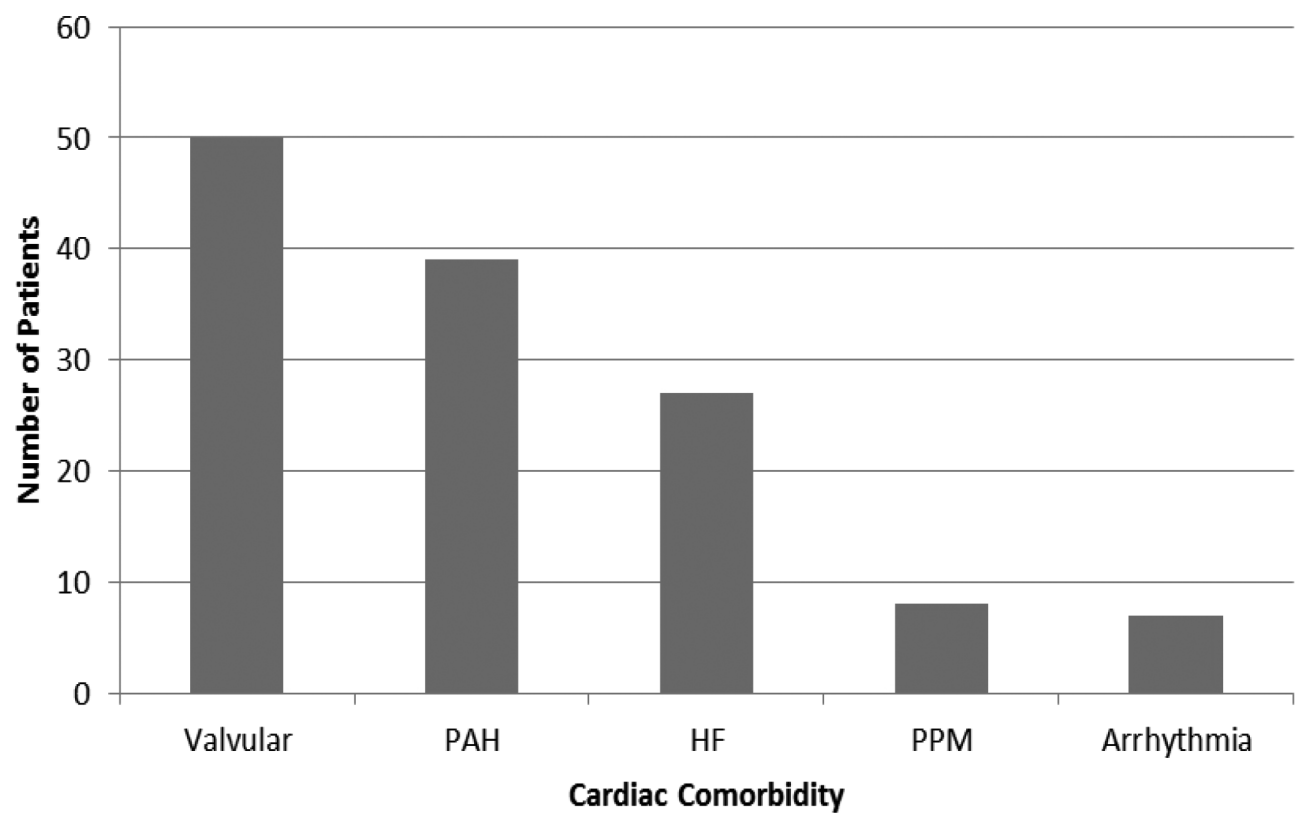

Abstract 72 Figure 2 T21 NORPAP Comorbidities. PAH Pulmonary Arterial Hypertension; HF Heart Failure; PPM Permanent Pacemaker 
Conclusion Although our numbers are small, the incidence of complications during pregnancy or immediately post partum are similar to those that we quote to our patients. Pregnant and post partum women complaining of severe chest pain should be brought to the immediate attention of senior staff and investigated urgently.

\section{\begin{tabular}{|l|l}
\hline 74 & NON-INVASIVE ASSESSMENT OF PULMONARY
\end{tabular} HAEMODYNAMICS IN FONTAN PATIENTS}

Aderonke Abiodun*, Luke Pickup, Heather Moore, Sarah Bowater, Sara Thorne, Lucy Hudsmith, Paul Clift. Queen Elizabeth Hospital Birmingham; *Presenting Author

10.1136/heartjnl-2016-309890.74

Introduction The Fontan circulation relies on a low pulmonary vascular resistance. At present the gold standard method of measuring this is with invasive cardiac catheterisation which can be difficult in this group. Previous work has shown that pulmonary vascular resistance is inversely correlated with pulmonary capacitance which can be approximated non-invasively using data derived from a maximal cardiopulmonary exercise test (CPEX). We have determined the non-invasive pulmonary artery capacitance (Ventilatory Product) in a large cohort of adult Fontan patients.

Methods 220 patients under regular follow up at University Hospital Birmingham were identified and CPEX results were available for 131 patients. We subdivided these into two groups, those with an atriopulmonary Fontan (AP) and combined those with lateral tunnel and total cavo-pulmonary connexion (TCPC). Parameters obtained were: NYHA functional class, maximum workload, ventilatory product, peak oxygen consumption, peak end tidal $\mathrm{CO}_{2}$ and $\mathrm{VE} / \mathrm{VCO}_{2}$ slope.

Results 77 patients with TCPC/lateral tunnel versus 54 AP Fontan were included. In the AP Fontan group, mean ventilatory product correlated positively with $\mathrm{pVO} 2\left(\mathrm{r}^{2}=0.344\right)$ and maximum workload $\left(\mathrm{r}^{2}=0.515\right)$ and negatively with $\mathrm{VE} / \mathrm{VCO}_{2}$ slope $\left(r^{2}=-0.366\right)$. In the combined TCPC/lateral tunnel Fontan group, mean ventilatory product correlated positively with pV02 $\left(\mathrm{r}^{2}=0.484\right)$ and maximum workload $\left(\mathrm{r}^{2}=0.485\right)$ and negatively with $\mathrm{VE} / \mathrm{VCO}_{2}$ slope $\left(\mathrm{r}^{2}=-0.127\right)$. When determined for each functional class ventilatory product was as follows in TCPC/lateral tunnel group: NYHA I $355.8+/ - \pm 100$, NYHA II $272.14 \pm 105$, NYHA III $241.76 \pm 90.3$. In the AP Fontan group mean ventilatory product was as follows: NYHA I $349.2 \pm 131.8$, NYHA II 271.5 121.5, NYHA III $273.9+/-140.6$. The mean VO2 peak was significantly higher in the combined TCPC/lateral tunnel group (26.64 vs 20.56, $\mathrm{p}=0.00)$. Further sub analysis within each functional class between both groups also showed statistical significance. TCPC/lateral NYHA class I mean V02 peak 29.56 vs 25.11 vs $(\mathrm{p}=0.01)$, class II mean V02 peak 22.72 vs $18(\mathrm{p}=0.038)$ and class III mean 22.14 vs 15.31 ( $\mathrm{p}=0.018$ ).

Discussion Invasive measurements of PVR are difficult in the Fontan patient. We have demonstrated that a non-invasive indirect measurement of pulmonary artery capacitance -the ventilatory product, correlates with measures of performance, and negatively correlates with $\mathrm{VE} / \mathrm{VCO}_{2}$, which is known to be of prognostic significance in heart failure. Furthermore our data shows that ventilatory product falls with increasing functional class. From this, we propose that the ventilatory product may be used to identify Fontan patients suitable for pulmonary vasodilator therapy. Our hypothesis however will require testing in prospective studies of pulmonary vasodilator therapy in Fontan patients.

\section{THE BURDEN OF AF AND STROKE IN ADULT CONGENITAL HEART DISEASE}

Aderonke Abiodun*, Heather Moore, Sayqa Arif, Sarah Bowater, Sara Thorne, Paul Clift, Lucy Hudsmith, Joseph De Bono. Queen Elizabeth Hospital Birmingham; *Presenting Author

\subsection{6/heartjnl-2016-309890.75}

Introduction Atrial arrhythmias are strongly associated with thromboembolism with 1 in 5 strokes being attributed to atrial fibrillation (AF). Patients with adult congenital heart disease (ACHD) are at an increased risk of developing atrial arrhythmias compared to those with structurally normal hearts either due to the inherent cardiac anomaly or due to scar formation from surgery. The $\mathrm{CHA}_{2} \mathrm{DS}_{2}$ VASC score is validated for stroke risk stratification for those with acquired heart disease. Its role in adult congenital heart disease however, is unclear.

Methods A retrospective review of the electronic ACHD database of all patients at a large quaternary specialist ACHD Centre with approximately 4500 patients under regular follow up. Data spanned from year 2000-2015 and data collected from referral letters, clinic letters, ECG and echocardiograms.

Results 376 patients with atrial arrhythmias were identified. Mean age was $57.7 \pm 15.8$ years with $51 \%$ female. 88 (23\%) of patients had complex disease (including those with a Fontan circulation) with the remainder having simple or moderate forms of congenital heart disease. In this cohort, 52 $(13.8 \%)$ patients had at least one thrombo-embolic event with $77 \%$ being either transient ischaemic attacks or strokes. The remainder of events were embolic peripheral artery occlusions, deep vein thromboses and pulmonary emboli. The mean age of first occurrence of thromboembolic event was $44.2 \pm 19$ years and mean $\mathrm{CHA}_{2} \mathrm{DS}_{2}$ VASC score was $1.04 \pm 0.7$ prior to the event. In $46 \%$ of cases the thromboembolic event occurred after the atrial arrhythmia had been diagnosed and $71 \%$ of patients were not anticoagulated at the time of their first event. Of those patients anticoagulated at the time of their event $(\mathrm{n}=9), 2$ patients had a documented subtherapeutic INR, 2 were non-compliant with their medication and 1 was on a Novel Oral Anticoagulant (NOAC). It was unclear in $11 \%$ of cases whether they were anticoagulated before the event. $88 \%$ of patients with a $\mathrm{CHA}_{2} \mathrm{DS}_{2}$ VASC score of 2 or more were anticoagulated, with $93 \%$ on warfarin and $5 \%$ on NOACs and $2 \%$ on low molecular weight heparin. Of those with a $\mathrm{CHA}_{2} \mathrm{DS}_{2}$ VASC score of $0-1,74 \%$ were on either warfarin or a NOAC. There were 4 known deaths from complications related to anticoagulation ( 2 gastrointestinal bleeds and 2 from massive haemoptysis).

Discussion Atrial arrhythmias are common in ACHD and there is a high incidence of stroke in those with AF. In those groups with higher incidence of $\mathrm{AF}$, loop recorders may be beneficial for detecting asymptomatic disease. The $\mathrm{CHA}_{2} \mathrm{DS}_{2}$ VASC scoring system does not appear to be applicable for stroke risk stratification in ACHD. The mean pre- event $\mathrm{CHA}_{2} \mathrm{DS}_{2}$ VASC was 1 . Another marker for patients with 